\title{
Diagnostic techniques for the carpal and fetlock joints in horses with arthritis')
}

\author{
MUSTAFA ARICAN, HANIFI EROL*, U. SAIT UCAN**, ÖZNUR KÖYLÜ*** \\ Department of Surgery, **Department of Microbiology, Faculty of Veterinary Medicine, University of Selcuk, Konya, Turkey \\ *Department of Surgery, Faculty of Veterinary Medicine, University of Erciyes, Kayseri, Turkey \\ ***Department of Biochemistry, Konya Education and Research Hospital, Konya, Turkey
}

\section{Arıcan M., Erol H., Ucan U. S., Köylü O. \\ Diagnostic techniques for the carpal and fetlock joints in horses with arthritis}

Summary

The aim of this study was to compare diagnostic techniques for the assessment of cartilage damage in the carpal and fetlock joints in horses with arthritis. The techniques compared were synovial fluid analysis, as well as radiographic, thermographic, ultrasonographic, and arthroscopic examinations. Sixteen horses of both sexes with arthritis, weighing $438 \pm 51 \mathrm{~kg}$ and at different ages were used as material. Before a detailed examination, an evaluation of the conformation and symmetry of the musculoskeletal system was performed. Nerve blocks definitively localized lameness to a specific site. Thermographic, radiographic, ultrasonographic, and arthroscopic examinations were performed after routine clinical examination. Synovial fluid and sera were collected for further analysis. In conclusion, although all examination techniques may yield useful information, their severe limitations were revealed, particularly in the detection of early articular cartilage damage. Arthroscopy is more reliable than other methods in cases of joint capsule lesions and intra-capsular lesions, as well as cartilage and synovial hyperplasia. Arthroscopy is considered to be advantageous and necessary.

Keywords: equine, thermograhy, radiography, arthroscopy, synovial fluid

Clinical examination of horses with arthritis is still very important. Imaging techniques, including radiography, thermography, ultrasonography, scintigraphy, computed tomography $(\mathrm{CT})$, and synovial fluid analysis, have long been established as non-invasive or minimally invasive diagnostic methods for the evaluation of chronic progressive joint diseases (20, 25). Arthroscopy is the diagnostic modality of choice for a direct assessment of the cartilage of the joint surface (17). Indentation instruments have been developed and used to judge the stiffness of cartilage during arthroscopic examination in order to evaluate its functional integrity $(1,16)$. Arthroscopy remains the gold standard for diagnosis of cartilage defects. Conventional synovial fluid analysis is still used to detect infection, but more recent work with synovial fluids and serum biomarkers offers real potential for diagnosis of early change in cartilage and bone $(21,25)$.

Degenerative arthritis, or osteoarthritis (OA), and the associated lameness is the most common cause of impaired athletic performance in horses (21). Early diagnosis of this joint disorder is essential for appro-

Financial support by Scientific Research Projects Coordination Unit of the Selçuk University (Project No. 09401021). priate therapeutic interventions and for prevention of further degeneration of articular cartilage (21). One of the first signs of incipient OA, before the appearance of macroscopically visible cartilage degradation, is the softening of the tissue, which is attributable mainly to the deterioration of the superficial collagen network and the depletion of proteoglycans $(2,6)$. Although osteochondral fragmentation, fractures, subchondral bone disease, osteoarthritis, and osteochondritis dissecans (OCD) are common in the horse, these diseases are usually diagnosed only after they have become established. Until recently, the detection of early or subtle disease has been poor, but the situation is improving. Clinical examination and radiographic imaging are still the most commonly used techniques for diagnosis of osteochondral disease, but osteochondral damage seen during arthroscopic surgery is usually more severe than that seen on radiographs $(7,8)$.

Arthroscopic examination of the fetlock joint may be indicated specifically as a diagnostic procedure or as part of an arthroscopic surgical procedure (18). Diagnostic arthroscopy is indicated in cases involving a lameness problem that has been localized to the fetlock, but for which radiographic signs are equivocal. 
The aim of the present study was to investigate the accuracy of diagnostic arthroscopy in the assessment of cartilage damage in the carpal and fetlock joints. One of the aims of the study was also to compare arthroscopic and thermographic imaging, as well as radiographic and ultrasonographic examinations. The relationship between biochemical and microbiological markers was investigated in the synovial fluid in osteoarthritis.

\section{Material and methods}

Sixteen horses with arthritis, weighing $438 \pm 51 \mathrm{~kg}$ and including both sexes and at various ages, were used as subjects. The study was carried out according to the criteria of the Ethical Committee of the Faculty of Veterinary Science at the University of Selçuk. The examination commenced after a precise history had been obtained to determine whether the onset had been sudden or insidious. The relationships between the presenting signs and exercise, medications received and shoeing were recorded. Before the detailed examination commenced, an evaluation of the conformation and symmetry of the musculoskeletal system was performed. Any swellings and muscle atrophy were reported. The horses were walked, initially on a hard surface, and note was taken of limb coordination and any indications of lameness. Finally, nerve blocks were used to localize the lameness definitively to a specific site.

Infrared thermography examination (IRT). Thermographic examinations of all animals were performed after a routine clinical examination. Images of joints were taken to determine their temperature. To adjust for skin (control) temperature, the difference between the affected area and the control area was calculated. Infrared images were taken of the dorsal view of the joints to monitor their temperature. IRT images were captured several times. All images were scanned with a hand-held portable infrared camera (Wahl, Thermal Imager HSI3000 Series), which was calibrated to the ambient temperature and absorptive conditions. The emissivity value selected in the camera before scanning was 0.93 . Generally, the dorsal surface of skin was cleaned of debris and moisture before images were taken. The horses were moved to a freestanding hoof-trimming stall, and, after visual examination of the hooves (10 to $20 \mathrm{~min}$ ), IRT images were taken. To reduce the effect of environmental factors on thermal data, all images were scanned at the same distance ( 1.5 to $2 \mathrm{~m}$ ) from the subject. The continuous output for each animal in each collection period was saved for 5 to $10 \mathrm{~s}$. The 'Wahl HSI3000 series imager' application was used for analysis of images previously saved on an HSI3000 SD card.

Radiological examination. Dorso-palmar, dorso-plantar, latero-medial and medio-lateral projections were used at $70 \mathrm{kV}$ and $30 \mathrm{mAs}$ for each joint (Sp-HF-4.0 Ralco Spain; Imago, Abbiategrasso, Milano; Regius Model 110 Konica, Minolta).

Ultrasonographic examination. Sonograhic examination was performed on full weight-bearing horses. Preparation included clipping and shaving the hairs, followed by antiseptic washing and rinsing with alcohol. Acoustic gel was applied to the carpal and fetlock region. A diagnostic ultrasound machine (Esaote Piemedical, Model 410477) with a 5 to $7.5 \mathrm{MHz}$ convex transducer was used to image bone and soft tissues in both sagittal and transverse planes. The most common indication for carpal ultrasound is regional swelling, wounds, or distension of carpal synovial structures. The dorsal surface of the fetlock was examined in both transverse and sagittal planes at the level of the sagittal ridge of the distal condoyle. There was often a little effusion. Images were printed using a video printer and thermosensitive paper.

Arthroscopic examination. Arthroscopic examination of the carpus and the palmar and plantar fetlock joints was performed using the dorsal approach, as described by McIlwraith (19). The joint was insufflated with $60 \mathrm{~mL}$ of isotonic saline and an arthroscope $\left(4 \mathrm{~mm}, 30^{\circ}\right.$ oblique lens, Lawton $\mathrm{GmbH} \& \mathrm{Co}$. KG) was inserted medial to the common digital extensor tendon by means of a blunt trocar. The arthroscopic findings were recorded on a Karl-StorzEndoscope, Telecam SL II).

The degree of chondropathy was assessed using the French Society of Arthroscopy scoring and grading systems (SFA) $(14,24)$. Briefly, the depth of cartilage lesions was classified on a 0 to 4 scale (6). Grade 0 indicates normal cartilage, grade 1 swelling and softening of the cartilage, grade 2 superficial fibrillation, grade 3 deep fibrillation down to subchondral bone, and grade 4 exposure of subchondral bone. The size of the lesions was estimated as a percentage of the whole articular cartilage that could be seen with the arthroscope.

Serum and synovial fluid analysis. Blood was collected from the jugular vein of each horse. Blood gases and hematologic parameters were investigated. Synovial fluid samples were collected using sterile needles and syringes and were transferred to vacutainer tubes. Synovial fluid was immediately centrifuged at $10,000 \mathrm{rpm}$ for 5 minutes to remove cellular debris. AlbG g/dl, AlkP U/L, ALT U/L, Amy U/L, AST U/L, BilD mg/dl, BiliT mg/dl, Ca mg/dl, Chol mg/dl, CK U/L, Cl mmol/dl, Crea mg/dl, DLDL mg/dl, GGT U/L, GLOB, GluC mg/dl, INBIL, Iron ug/dl, K mmol/dl, LD U/L, Lip U/L, Mg mg/dl, Na mmoldl, Phos mg/dl, TP g/dl, Trig mg/dl, UHDL mg/dl, UIBC ug/dl, Urea mg/dl and Urate $\mathrm{mg} / \mathrm{dl}$ were measured in serum and synovial fluid. Physical, biochemical, and cytological properties of synovial fluid from arthritic joints were investigated. Synovial fluid was obtained by arthrocentesis of 16 joints. The following parameters were recorded: appearance of synovial fluid and volume at the time of arthrocentesis, signs of excessive effusions, manifestations of joint pain or impairment of gait, gross or palpable enlargements of carpal and fetlock joints, and evidence of systemic diseases. Rheumatoid factors (CRP, RF, ASL) were assayed in the sera and synovial fluids. Mucinous precipitation was recorded as normal, tight, fair, poor, or very poor.

Microbiological analysis. Joint fluid was also collected for laboratory analysis to look specifically for signs of infection. Synovial fluid was collected aseptically into sterile tubes for aerobic and anaerobic bacterial culture on solid agars at the microbiology laboratory of the faculty. Standard conventional culture methods were used for the aerobes and anaerobes that might be causatives of the equine synovitis $(9,22)$. Two plates of Columbia agar (CM 0331, 
Oxoid) with $5 \%$ defibrinated sheep blood and a plate of MacConkey agar (CM 0007, Oxoid) were each inoculated with one drop $(\sim 25 \mu \mathrm{L})$ of synovial fluid. The plates (one Columbia agar and one MacConkey agar) were incubated aerobically at $37^{\circ} \mathrm{C}$ for at least 4 days. Another Columbia agar plate was incubated anaerobically at $37^{\circ} \mathrm{C}$ for the same period, using the BBL GasPak EZ Anaerobe Pouch System (260683, BBL). All the plates were inspected daily. If no

Tab. 1. Termographic results for carpal (C) and metacarpal (MC) joints

\begin{tabular}{|r|c|c|c|c|c|}
\hline Horses & Joints & $\begin{array}{c}\text { Dorso-palmar } \\
\text { heat increase }\end{array}$ & $\begin{array}{c}\text { Latero-medial } \\
\text { heat increase }\end{array}$ & $\begin{array}{c}\text { Medio-lateral } \\
\text { heat increase }\end{array}$ & $\begin{array}{c}\text { Palmar-dorsal } \\
\text { heat increase }\end{array}$ \\
\hline 1 & C & + & + & + & + \\
2 & C & + & + & + & + \\
3 & MC & + & + & + & + \\
4 & MC & + & + & + & + \\
5 & MC & + & + & + & - \\
6 & C & - & - & - & - \\
7 & C & + & + & - & - \\
8 & MC & - & - & - & - \\
9 & MC & + & + & + & + \\
10 & C & + & - & - & - \\
\hline 11 & MC & + & - & + & - \\
12 & C & + & - & + & - \\
13 & MC & - & + & + & + \\
14 & MC & + & + & + & + \\
15 & C & + & + & + & + \\
16 & C & + & + & + & + \\
\hline
\end{tabular}

Explanations: + Indicates a positive result; $\mathrm{C}$ - carpal joints; $\mathrm{MC}$ - metacarpal joints

Tab. 2. Radiological results for carpal (C) and metacarpal (MC) joints

\begin{tabular}{|c|c|c|c|c|c|c|c|}
\hline Horses & Joints & $\begin{array}{l}\text { Contraction } \\
\text { of the joint } \\
\text { surface }\end{array}$ & $\begin{array}{l}\text { Osteophyte } \\
\text { production }\end{array}$ & $\begin{array}{l}\text { Subchondral } \\
\text { sclerosis }\end{array}$ & $\begin{array}{c}\text { Subchondral } \\
\text { cyst } \\
\text { formation }\end{array}$ & Subluxation & $\begin{array}{l}\text { Increase } \\
\text { in synovial } \\
\text { fluids }\end{array}$ \\
\hline 1 & C & - & - & - & + & - & - \\
\hline 2 & C & - & - & - & - & - & - \\
\hline 3 & MC & - & - & - & - & + & \\
\hline 4 & MC & + & - & - & - & - & - \\
\hline 5 & MC & + & - & - & - & - & - \\
\hline 6 & C & - & - & - & - & - & - \\
\hline 7 & C & - & - & - & - & - & - \\
\hline 8 & MC & + & - & - & - & - & - \\
\hline 9 & MC & - & + & - & - & - & - \\
\hline 10 & C & - & + & - & - & - & - \\
\hline 11 & MC & - & + & - & - & - & - \\
\hline 12 & C & - & - & - & - & - & - \\
\hline 13 & MC & + & - & - & - & - & - \\
\hline 14 & MC & - & - & - & - & - & - \\
\hline 15 & C & + & - & - & - & - & - \\
\hline 16 & C & - & - & - & - & - & - \\
\hline
\end{tabular}

Explanations: as in Tab. 1. growth was visible by the $4^{\text {th }}$ day of incubation, the culture was regarded as negative.

Data analysis. A t-test was performed for each category at each level to detect any significant differences in the number values assigned for each category for synovial fluids analysis. In thermographic imaging, a positive or negative evaluation was made according to the temperature difference between the hottest region and the coldest region.

\section{Results and discussion}

Lameness was localized in each case to a specific site by detailed physical examination combined with with nerve blocks.

Infrared thermography. In cases of arthritis, the computer software of the infrared thermal camera showed temperature in suspected regions to be increased by 0.5 to $1.5^{\circ} \mathrm{C}$ compared with that in the normal regions. Thermography findings showed that the horses with arthritis numbered 1, 2, 3, 4, $9,10,11,12$, and 13 had an increased temperature of the joints, which was not recorded during palpation.

Radiology. Radiographs did not demonstrate cartilage damage. Radiology and clinical examination diagnosed only functional decline of the joints in horses $1,4,5,8,13$, and 15 , and contraction of the joints with surface osteophytes in 9, 10, and 11. Number 5 showed subluxation of the joint. The results of the radiological examination are given in Table 2.

Ultrasonograpy. Some of the cases required distension to detect the relative degree of effusion versus synovial thickening. Hypertrophy of the synovial membrane, especially villonodular synovitis, was recognized as a moderately echogenic soft tissue mass. It was difficult to examine articular cartilage because it was hidden by the adjacent bone. The cartilage forms a sharply defined hypoechoic rim superficial to bone. Its surface is normally smooth, as is the surface of the underlying bone. In the fetlock joint, in some cases, villonodular synovitis was recognised as a discrete, moderately echogenic mass with regular to irregular margins. Ultrasound examination of horses 1, 6, 14, and 15 showed increased effusion; for example, ultrasonographic images of the dorsal aspect of the 
fetlock joint revealed that the joint capsule appeared thick with a moderately homogeneous echoic structure. The dorsal aspect of the subchondral bone of the sagital ridge and the proximal phalanx were seen as regular and well defined lines. The dorsal and proximal palmar synovial recesses of the fetlock joint contained a small amount of anechoic synovial fluid. Joints in horses 5, 7,8 , and 14 did not show any evidence of problems. The results of ultrasonography are given in Table 3 .

Arthroscopy. Arthroscopic examination showed synovitis, erosion of cartilage, and osteophytes in horses $1,5,6,7,8,14$, and 15 , in which lesions of degenerative joint disease were diagnosed. Synovial fluid distension and synovial membrane thickening or proliferation are indicative of recent or chronic arthritis. These joints showed no changes on radiographic or ultrasonographic examination, but clinical findings were sufficient to encourage the clinician to look for a causative pathological process in the joint. In the majority of clinical cases, synovial lesions are observed with lesions of the other components of the joint. The results of arthroscopy are given in Table 4.

Synovial fluid examination. The synovial fluid was pale yellow, clear, free of flocculent material, and did not clot. Its volume varied in direct proportion to the size of the individual carpal joint. Relative viscosity was related to volume, polymerization, protein concentration, and the quantity of hyaluronic acid. The quality of the mucinous precipitate was uniformly high. These investigations indicated that inflammation of the syno-
Tab. 3. Ultrasonographic results for carpal (C) and metacarpal (MC) joints

\begin{tabular}{|c|c|c|c|c|}
\hline Horses & Joints & Joint effusion & $\begin{array}{c}\text { Synovial } \\
\text { hypertrophy }\end{array}$ & $\begin{array}{c}\text { Cartilage } \\
\text { structures }\end{array}$ \\
\hline 1 & C & + & - & - \\
2 & C & + & - & - \\
3 & MC & - & - & - \\
4 & MC & - & - & - \\
\hline 5 & MC & - & - & - \\
\hline 6 & C & + & - & - \\
\hline 7 & C & - & - & - \\
\hline 8 & MC & - & - & - \\
\hline 9 & MC & - & - & - \\
\hline 10 & C & + & - & - \\
\hline 11 & MC & + & - & - \\
\hline 12 & C & + & + & - \\
\hline 13 & MC & + & - & - \\
\hline 14 & MC & - & - & - \\
\hline 15 & C & + & - & - \\
\hline 16 & C & + & - & - \\
\hline
\end{tabular}

Explanations: as in Tab. 1.

vial membrane and articular degeneration are important factors in many common joint disorders and contribute significantly to joint dysfunction. Information gained through synovial fluid analysis can indicate the nature and extent of intra-articular pathologies and is a valu-

Tab. 4. Diagnosis of osteoarthritis and synovitis of joints assessed

\begin{tabular}{|c|c|c|c|c|c|c|c|c|c|c|c|}
\hline \multirow{3}{*}{ Horses } & \multirow{3}{*}{ Joints } & \multirow{3}{*}{$\begin{array}{c}\text { DJD } \\
\text { Osteoarthritis }\end{array}$} & \multicolumn{9}{|c|}{ Inflamatory Joint Diseases } \\
\hline & & & \multirow[t]{2}{*}{ RA } & \multicolumn{8}{|c|}{ Synovitis } \\
\hline & & & & Hyperemia & Odema & $\begin{array}{c}\text { Petechial } \\
\text { hemorrhage }\end{array}$ & $\begin{array}{l}\text { Hyper. } \\
\text { villus. }\end{array}$ & $\begin{array}{c}\text { Villial } \\
\text { thickness }\end{array}$ & $\begin{array}{l}\text { New villial } \\
\text { formation }\end{array}$ & $\begin{array}{l}\text { Vilial } \\
\text { atrophy }\end{array}$ & $\begin{array}{c}\text { Polipoid } \\
\text { Villial } \\
\text { Formation }\end{array}$ \\
\hline 1 & C & + & - & + & - & - & + & + & + & - & - \\
\hline 2 & C & - & - & + & + & - & + & + & + & - & - \\
\hline 3 & MC & - & - & - & + & + & - & + & + & - & - \\
\hline 4 & MC & - & - & + & + & - & + & - & - & - & - \\
\hline 5 & MC & + & - & - & + & - & - & + & - & + & + \\
\hline 6 & C & + & - & + & - & - & - & + & + & - & - \\
\hline 7 & C & + & - & + & - & - & - & + & & - & - \\
\hline 8 & MC & + & - & + & - & - & - & + & + & - & - \\
\hline 9 & MC & - & - & + & - & - & + & + & + & - & - \\
\hline 10 & C & - & - & + & - & - & - & + & + & - & - \\
\hline 11 & MC & - & - & + & - & - & - & + & - & - & - \\
\hline 12 & C & - & - & + & - & - & - & + & + & - & - \\
\hline 13 & MC & + & - & + & - & - & + & + & + & + & - \\
\hline 14 & MC & + & - & + & + & + & + & + & + & - & - \\
\hline 15 & C & & - & + & - & - & - & + & + & - & - \\
\hline 16 & C & + & - & + & + & + & + & + & + & - & - \\
\hline
\end{tabular}

Explanations: + Indicates a positive result; $\mathrm{C}$ - carpal joints; $\mathrm{MC}$ - metacarpal joints; DJD - degenerative joint diseases; RA - rheumatoid arthritis 
able adjunct to other diagnostic techniques in establishing diagnoses, selecting treatments, and developing realistic prognoses.

Variations in joint fluid parameters were shown to be useful in differentiating a normal joint from such disorders as traumatic effusion acute, chronic, and leukocyte synovitis. The most helpful synovial fluid parameters in this study were relative viscosity, hyaluronic acid, mucin clot, protein, leukocyte count, and cartilage fragments. $\mathrm{Cl}(\mathrm{mmol} / \mathrm{dl})$, Crea $(\mathrm{mg} / \mathrm{dl})$, and Urea $(\mathrm{mg} / \mathrm{dl})$ levels $(\mathrm{p}<0.05)$ showed correlations in serum and synovial fluid. Escherichia coli, Klebsiella ssp., Salmonella ssp., Staphylococcus aureus, piyogenic/faecal streptococci, Actinobacillus equuli and Rhodococcus equi were not detected in the synovial fluid samples.

Medical thermography is a noninvasive technique. It displays the superficial body temperature in order to detect inflamed tissue and is especially useful in diagnosing lameness in horses and in localizing lesions $(4,12,23)$. In this study, infrared thermography facilitated the finding of region(s) of temperature differences in horses with arthritis: $56.25 \%$ of all animals studied showed increased temperature in the joints. These changes were not recorded during palpation. The temperature may depend on the stage of disease. Thermography should be used for early diagnosis in combination with other diagnostic imaging methods $(4,12,19,23)$.

Radiographic examination is still the most widely used imaging technique for the diagnosis of osteochondral disease, but it is an insensitive method of diagnosis (14). Articular cartilage cannot be viewed radiographically, except when there is extensive loss and decreased joint space, and 30 to $40 \%$ change in bone mineral density is required before bone changes can be observed (24). In this study, radiographs were incapable of demonstrating cartilage damage. Radiology showed only that $37.5 \%$ of the joint surfaces had contraction of the joints, and $18.75 \%$ of the joints showed osteophyte formation. One animal showed subluxation of the joint. The cases were presented to the clinic early in the disease, so radiological changes were difficult to see. McIlwraith (19) showed that multiple images are required for evaluation of a three-dimensional structure. Disease is often recognized after significant damage has occurred, and this lack of sensitivity can prevent early and accurate diagnosis. Measuring the joint space is fraught with error (24). The significance of osteophytes is frequently unrelated to intra-articular pathological change, and considerable change in bone density is necessary to identify sclerosis and erosion. Widmar and Blevins (24) found that radiographs were insensitive in detecting subchondral bone sclerosis and erosion when compared with histology. It has also been pointed out that superimposition of osteophytes may appear as sclerosis (14). Therefore we did not detect any problems by X-ray.
Ultrasonographic examination of joints was pioneered by Denoix (10). A linear transducer operating at $8 \mathrm{mHz}$ and a convex transducer at 5 to $7.5 \mathrm{mHz}$ are typically used for the fetlock and palmar/plantar metacarpal/tarsal regions. Like every imaging technique, ultrasound examination of joints has some limitations, which are related mainly to anatomical particularities of the joints. For example, in the fetlock joint the main limitation of ultrasonography is the inability to image the proximal articular surface of $\mathrm{P} 1$ and the palmar (plantar) articular surface of the metacarpal (matatarsal) condyle. In the carpus, the intra-articular ligaments and most of the articular surfaces cannot be imaged. The technique can, however, be used to evaluate soft tissues associated with the joint, including the collateral ligaments, joint capsule, other associated ligaments, and the menisci (11). In the present study, joint effusion was demonstrated in cases of arthritis. Hypertrophy of the synovial membrane, especially villonodular synovitis, was recognized as a moderately echogenic soft tissue mass. However, we also found during the examination that it was difficult to visualize the articular cartilage because it was hidden by the adjacent bone. Abnormal echogenicity of the underlying bone is indicative of periarticular bone lysis.

Synovial fluid and serum parameters are used as biomarkers because cartilage degradation involves destruction of the collagen framework and loss of proteoglycan (failure of synthesis) products of type II collagen; proteoglycans are liberated in increasing concentrations into the synovial fluid and ultimately the serum. Some biochemical parameters were shown to be correlated in serum and joint fluid: $\mathrm{Cl}$, Crea, and urea. Creatinine elevation was asymptomatic in these cases, and asymptomatic reversible impairment of renal function in cases of arthritis has previously been reported (15). Elevated CPK may be caused by disease or injury affecting the heart muscle, skeletal muscle, or brain. Higher levels of CPK can also be seen after trauma or intramuscular injections and in cases of muscle disease. Urea concentrations in joint fluid were directly proportional to those in serum throughout a wide range of concentrations in normal joints. From this relationship, the dilution factor introduced by joint fluid can be determined. Urea provides a robust method of quantifying and correcting for the dilution of synovial fluid due to joint lavage or inflammation (5). Fluid biomarkers can be divided into synthetic and degradative markers, and both are useful in diagnosing early change in cartilage and bone (13). Clinical studies have demonstrated the usefulness of serum biomarkers in diagnosing early equine joint disease $(9,22)$, defining the clinical significance of OCD.

The most common microorganisms cultured from equine synovial fluid samples are Escherichia coli, Klebsiella ssp., Salmonella ssp., Staphylococcus aureus, pyogenic/faecal streptococci, Actinobacillus 
equuli, and Rhodococcus equi (9). Culturing a causative organism is the gold standard means of diagnosing synovial infection as long as no delay (usually 48 hours) has been allowed from sample submission to diagnosis (22). No microorganisms were detected in the present study.

Arthroscopy is now considered part of routine equine surgery and has, for the most part, replaced arthrotomy (19). It allows better visualisation of joint anatomy and inflicts less damage on the joint capsule and surrounding tissues, leading to quicker return to use and a more favourable outcome for the horse. In this study, arthroscopic examination included all the joints. Synovitis, erosion of cartilage, and osteophytes were seen in $43.75 \%$ of the joints, and degenerative joint disease lesions were also diagnosed. Synovial fluid distension and synovial membrane thickening or proliferation are indicative of recent or chronic arthritis. In our study, complete arthroscopic examination of the metacarpophalangeal or metatarsophalangeal joints was not possible, as supported by earlier studies (17). Two arthroscopic approaches were required to achieve as thorough an examination as possible (17). It is still considered the gold standard for diagnosing equine joint problems. Joint pathology, such as meniscal and crucial lesions, has been described using this technique, but such lesions are not detectable by radiography or other diagnostic techniques. Another advantage of the use of arthroscopy is the potential benefit of lavage $(3,14,19)$.

In summary, radiography is important in the diagnosis of joint diseases, but provides limited information on the articular cartilage, synovial membrane, and intra-articular ligaments. Thermographic examination is an important method that can assist in obtaining a diagnosis when combined with other diagnostic imaging methods and clinical examination, rather than being an independent, alternative technique. Artefacts expected to occur should be taken into consideration before thermographic examination is conducted, and an appropriate environment and patient preparation are required. Ultrasonography is recognized as an important diagnostic tool, especially in cases with synovial effusion. Degenerative joint diseases, articular cartilage lesions, and the increase in the amount of synovial fluid relative to the protein concentration are emphasized. Lesions inside the joint, including those of the synovial membrane, require arthroscopic examination and treatment.

In conclusion, although all examination techniques may yield useful information, they also have important limitations, particularly in the detection of early damage to the articular cartilage. Arthroscopy is more reliable for detection of joint capsule and intra-capsular lesions, as well as cartilage and synovial hyperplasia than other methods. Arthroscopy is considered to be advantageous and necessary.

\section{References}

1. Appleyard R. C., Swain M., Khanna S., Murell G. A. C.: The accuracy and reliability of a novel handheld dynamic indentation probe for analyzing articular cartilage. Phys. Med. Biol. 2001, 46, 541-550.

2.Arokoski J. P., Jurvelin J. S., Väätäinen U., Helminen H. J.: Normal and pathological adaptations of articular cartilage to joint loading. Scand J. Med. Sci. Sports. 2000, 10, 186-198.

3. Auer J., Stick J.: Equine Surgery. Saunders, Elsevier 2011, p. 1054-1073.

4. Bathe A. P.: Thermography, [in:] Floyd A. E., Mansmann R. A. (ed.): Equine Podiatry, Saunders, St. Louis 2007, p. 167-170.

5. Billinghurst R. C., Brama P. A., Vance P. A. J., Van Weeren P. R., Knowlton M. S., Mcllwraith C. W.: Evaluation of serum concentrations of biomarkers of skeletal metabolism and results of radiography as indicators of severity of osteochondrosis in foals. Am. Vet. Med. Ass. 2004, 65, 143-150.

6. Brommer H., Rijkenhuizen A. B. M., Brama P. A. J., Barneveld A., Van Weeren P. R.: Accuracy Of Diagnostic Arthroscopy For The Assessment Of Cartilage Damage In The Equine Metacarpophalangeal Joint. Equine Vet. J. 2004, 36, 331-335.

7. Brommer H., Van Weeren P. R., Brama P. A. J.: New approach for quantitative assessment of articular cartilage degeneration in horses with osteoarthritis. Am. Vet. Med. Ass. 2003, 64, 83-87.

8. Brommer H., Van Weeren P. R., Brama P. A. J., Barneveld A.: Quantification and age-related distribution of articular cartilage degeneration in the equine fetlock joint. Equine Vet. J. 2003, 35, 697-701.

9. Carter G. R.: Diagnostic Procedures in Veterinary Bacteriology and Mycology. Charles C. Thomas Publisher, USA 1984, p. 1-515.

10. Denoix J. M.: Ultrasonographic examination in the diagnosis of joint disease, [in:] McIlwraith C. W., Trotter G. W. (ed.): Joint disease in the horse. Saunders, Philadelphia 1996, p. 165-202

11. Driver A. J., Barr F. J., Fuller C. J.: Ultrasonography of the medial palmar intercarpal ligament in the Thoroughbred: Technique and normal appearance. Equine Vet. J. 2004, 36, 402-408.

12. Eddy A. L., Van Hoogmoed L. M., Snyder J. R.: The role of thermography in the management of equine lameness. Veterinary J. 2001, 162, 166-167.

13. Frisbie D. D., Al-Sobayil F., Billinghurst R. C., Kawcak C. E., McIlwraith $C$. $W$ : Changes in synovial fluid and serum biomarkers with exercise and early osteoarthritis in horses. Osteoarthritis Cartilage 2008, 16, 1196-1204.

14. Greenfield G.: Analytical approach to bone radiology, [in:] Greenfield G. (ed.): Radiology of Bone Diseases 7. JB Lippincott, Philadelphia, In equine surgery and lameness. Philadelphia W.B. Saunders 1998, p. 643-654.

15. Kraus V. B., Huebner J. L., Fink C., King J. B., Brown S., Vail T. P., Guilak F: Urea as a passive transport marker for arthritis biomarker studies. Arthritis Rheumatol. 2002, 46, 420-427.

16. Lyyra T: Development, validation and clinical application of indentation technique for arthroscopic measurement of cartilage stiffness. Doctoral diss., Kuopio University, Finland 1997

17. McIlwraith C. W.: Diagnostic and Surgical Arthroscopy in the Horse. Lea\&Febiger, London 1990, p. 180-392.

18. McIlwraith C.W.: Evaluation of serum concentrations of biomarkers of skeletal metabolism and results of radiography as indicators of severity of osteochondrosis in foals. Am. J. Vet. Res. 2004, 65, 143-150.

19. McIlwraith C. W.: Recent Advances in Diagnosis of Equine Joint Disease. Proc. 2010 Kentucky Equine Research Nutrition Conference, 26-27 April, 2010

20. Park R. D., Steyn P. F., Wrigley R. H.: Imaging techniques in the diagnosis of equine joint disease, [in:] McIlwraith C. W., Trotter G. W. (ed.): Joint disease in the horse. Saunders, Philadelphia 1996, p. 145-164.

21. Price J.: A report from a workshop on molecular markers of cartilage and bone metabolism in the horse. Equine Veterinary Education 2001, 14, 6-11.

22. Smith M. R. W.: Equine synovial fluid: synoviocentesis and analysis, Proc. $49^{\text {th }}$ British Equine Veterinary Association Congress, 7-10 $0^{\text {th }}$ September, Liverpool, UK 2010.

23. Turner T. A.: Diagnostic thermography. Veterinary Clinics; Equine 2001, 17, 5-113.

24. Widmar W., Blevins $W$ : Radiographic evaluation of degenerative joint disease in horses: Interpretive principles. Compendium on Continuing Education for the Practicing Veterinarian 1994, 16, 907-918

25. Wimer C. L.: Characterization of the equine intra-articular inflammatory response to common diagnostic and treatment procedures. Residents Forum. ACVS Annual Convention. San Antonio Texas 2013.

Corresponding author: Dr. Mustafa Arican, Department of Surgery, Faculty of Veterinary Medicine, The University of Selcuk, Campüs, Konya, Turkey; e-mail: marican@selcuk.edu.tr 